\title{
Caracterización de los servicios farmacéuticos de atención primaria del Sistema Único de Salud en Londrina, Paraná, Brasil
}

\author{
Poliana Vieira da Silva Menolli, ${ }^{1}$ Adriana Mitsue Ivama ${ }^{2}$ \\ y Luis Cordoni Júnior ${ }^{3}$
}

Forma de citar Menolli PVS, Ivama AM, Cordoni L Jr. Caracterización de los servicios farmacéuticos de atención primaria del Sistema Único de Salud en Londrina, Paraná, Brasil. Rev Panam Salud Publica. 2009:25(3): 254-9.

RESUMEN Objetivos. Caracterizar a los servicios farmacéuticos de atención primaria del Sistema Único de Salud (SUS) de Brasil.

Métodos. El estudio se llevó a cabo en Londrina, Paraná, Brasil, de enero a marzo de 2003. Se utilizaron los indicadores de uso de los medicamentos propuestos por la Organización Mundial de la Salud. Se incluyeron 13 unidades de atención primaria de salud, donde se entrevistaron 390 pacientes.

Resultados. Ninguna de las unidades contaba con un farmacéutico. El promedio de duración de la consulta médica fue de 8,6 minutos; el de medicamentos por prescripción, 2,2; y el de uso de inyectables, 10,9\%. La denominación genérica se utilizó en 66,5\% de las prescripciones y 73,3\% de los medicamentos constaban en la Lista Municipal de Medicamentos Esenciales (REMUME). Solamente $46,6 \%$ de los pacientes entrevistados refirieron tener la información minima para usar correctamente los medicamentos prescritos.

Conclusión. Estos resultados pueden contribuir para la reorganización de los servicios farmacéuticos en el municipio, ya que señalan la necesidad del fortalecimiento y del uso racional de medicamentos como recursos terapéuticos necesarios para apoyar a las acciones de salud.

Palabras clave Medicamentos, farmacoepidemiología, servicios farmacéuticos, medicamentos esenciales, atención primaria, Brasil.

En el contexto de la salud, los desafíos del acceso a servicios y a insumos en el marco de la integralidad de la atención a la salud son enormes. La garantía del acceso, de la calidad de la atención y de la

\footnotetext{
Universidade Estadual do Oeste do Paraná (UNIOESTE). La correspondencia se debe dirigir a Poliana Vieira da Silva Menolli, Calle Academia, 695 Barrio Universitário, Cascavel, Paraná, Brasil CP 85819-100. Correo electrónico: polianacas cavel@hotmail.com

2 Agência Nacional de Vigilância Sanitária (Anvisa). Correo electrónico: adriana.ivama@anvisa.gov.br; Adriana.ivama@gmail.com

3 Universidade Estadual de Londrina (UEL). Correo electrónico: cordoni@sercomtel.com.br
}

seguridad de los pacientes son temas que están en la agenda de salud de una buena parte de los países de la Región de las Américas. De manera simultánea a las reformas del sector salud y a las restricciones presupuestarias, se verifica la pérdida de la centralidad de los Estados Nacionales y el aumento del protagonismo de los actores supranacionales y las empresas transnacionales en el contexto de la globalización. Para el sector farmacéutico, el incremento de empresas transnacionales se acompaña de la renovación y la comercialización cada vez más rápidas de tecnologías de salud, no siempre costo-efectivas o necesarias, lo que genera gran presión para su incorporación en los sistemas de salud.

La importancia del uso racional de medicamentos como parte de la calidad de los servicios de salud es indudable, ya que corresponden al recurso terapéutico más utilizado; además, los gastos farmacéuticos representan el segundo mayor gasto en los sistemas de salud, después del de personal (1).

De acuerdo con la Organización Mundial de la Salud (OMS), el gasto en medicamentos en los países en desarrollo varía de $24 \%$ a $66 \%$, y en países desarro- 
llados, de $7 \%$ a $30 \%$ del gasto total en salud. En el caso de los servicios farmacéuticos, se recomienda el fortalecimiento de las políticas, de la gestión y de la atención a los pacientes (2).

La necesidad de evaluar la calidad de la asistencia en salud es de interés mundial, principalmente porque se entiende que una buena práctica terapéutica implica una buena atención a la salud (3). Frente a los problemas que el uso irracional de medicamentos ocasiona tanto a los individuos como a los sistemas de salud, la OMS y otros autores desarrollaron indicadores para estudiar dicho uso $(4,5)$. La finalidad de estos indicadores es definir un número limitado de parámetros que permitan describir la situación de un país, región o centro de salud, en lo que se refiere al uso de medicamentos.

En el Brasil, la Constitución de 1988 estableció la creación del Sistema Único de Salud (SUS), con los principios de acceso universal y gratuito, atención integral y un nivel más amplio de participación de la sociedad, teniendo como base los modelos de protección social (6). Asimismo, el SUS prevé el acceso a los servicios farmacéuticos como parte de la integralidad de las acciones de salud. El SUS es un sistema descentralizado y los gestores del sistema son el Ministerio de Salud, las secretarías estaduales de salud y las secretarías o autarquías municipales de salud, que están integrados pero cada uno tiene cierto grado de autonomía.

Los servicios farmacéuticos del sector público eran prácticamente, hasta hace poco, sinónimo de distribución de medicamentos. Los municipios tenían un papel muy limitado: el de distribuir los medicamentos adquiridos por el gobierno federal de forma centralizada, sin considerar las particularidades de cada parte del país (7). Con el cambio del modelo de atención de salud, la descentralización y la consecuente reorganización de los servicios del SUS, los servicios farmacéuticos asumieron otra dimensión. Un marco importante fue la aprobación de la Política Nacional de Medicamentos (PNM) en 1998, que propuso la adopción de la Relación Nacional de Medicamentos Esenciales (RENAME), la reorientación de los servicios farmacéuticos mediante su fortalecimiento en el nivel primario y la promoción del uso racional de medicamentos. En los años noventa, hubo una fuerte inversión en la capacitación para la gestión de los servicios far- macéuticos, con incentivos para su organización en los estados y municipios (8), así como una definición más clara de responsabilidades para la coparticipación en la financiación de los medicamentos en la atención primaria por el Ministerio de Salud y las secretarías de salud de estados y municipios.

Al final de la década de 1990, hubo una gran cantidad de medicamentos falsificados, lo que provocó la creación de la Agência Nacional de Vigilância Sanitária (Anvisa) y el fortalecimiento de la regulación sanitaria de medicamentos. En 2003, como parte de su reorganización, el Ministerio de Salud creó la Secretaria de Ciência, Tecnologia e Insumos Estratégicos y, como parte de ella, el Departamento de Assistência Farmacêutica e Insumos Estratégicos. Este departamento, aparte de ser el gestor de la Política Nacional de Medicamentos, también es el gestor del presupuesto de medicamentos del Ministerio de Salud y del componente de medicamentos de los programas de salud pública, en coordinación con los gestores de estos programas; antes estas acciones se realizaban de forma fragmentada. En 2004, se aprobó la Política Nacional de Servicios Farmacéuticos, que incorpora los referenciales para la calificación de los servicios, considerando la atención farmacéutica y el fortalecimiento del uso racional de medicamentos (9).

El municipio de Londrina está localizado al norte del Estado de Paraná, en la región sur del país. Tiene una población de 447065 habitantes y es el municipio polo de una región metropolitana de siete municipios que suman el total de 662885 habitantes; también es referencia para un área de influencia estimada en 4,5 millones de habitantes (10). Fue uno de los primeros municipios del país en realizar el fortalecimiento de la red de atención primaria, en la década de 1970.

Cuando se llevó a cabo la investigación, la red de unidades de atención básica (UBS), equivalentes a unidades de atención primaria, tenía 51 unidades en Londina, 38 de ellas en la zona urbana. La responsabilidad por el sistema local de salud del municipio es de la Autarquia Municipal de Saúde (AMS). En el mismo período, el municipio contaba con una central de abastecimiento responsable por el suministro de medicamentos a las farmacias hospitalarias del SUS y "dispensarios" de medicamentos en las unidades básicas de salud; también había farmacias privadas, pero no se incluyeron en el estudio.

La evaluación de los servicios farmacéuticos en la atención primaria es una estrategia gubernamental prevista en la PNM (9). Congruente con esta estrategia, el objetivo de esta investigación fue caracterizar los servicios farmacéuticos en la atención primaria del SUS en Londrina, Paraná, Brasil.

Esta investigación se realizó como parte de los requisitos para obtención del grado de "Mestre" en Salud Colectiva del Programa de Salud Colectiva de la Universidade Estadual de Londrina, con beca de la Coordenação de Aperfeiçoamento de Pessoal de Educação Superior (CAPES).

\section{MATERIALES Y MÉTODOS}

Se llevó a cabo un estudio transversal descriptivo, no controlado, con base en la metodología propuesta por la OMS (4). Se siguieron las normas nacionales de ética en investigación con seres humanos y el proyecto fue aprobado por el Comité de Ética en Investigación de la Universidad Estadual de Londrina. Se obtuvo el consentimiento fundamentado de los participantes y la autorización formal de los responsables de las unidades donde se realizó la investigación.

La muestra fue estratificada y definida por sorteo; las 13 unidades estudiadas eran parte de las 38 unidades localizadas en el área urbana. Tal selección atendió a dos criterios: la capacidad de las UBS, definida por la Secretaría Municipal de Salud según las dimensiones del servicio y su productividad; y la localización geográfica, para garantizar la diversidad y la presencia de todas las regiones urbanas en el muestreo de las unidades (Norte, Sur, Este, Oeste y Central).

Las visitas para la recolección de datos se realizaron de enero a marzo de 2003. Se incluyeron 390 pacientes (30 en cada UBS, 15 de pediatría y 15 de clínica general); después de las consultas médicas, las cuales se cronometraron, se entrevistaron a los pacientes y se revisaron sus historias clínicas, que contenían la prescripción de medicamentos. Como parte de la estandarización del funcionamiento de los servicios en el municipio, solamente se ofrecían recetas a los pacientes cuando el medicamento no estaba disponible en el servicio y había que adquirirlo en una farmacia privada.

Los instrumentos propuestos por la OMS (4) se adaptaron específicamente 
para este fin: se tradujeron al portugués y se realizó una prueba preliminar (pretest) en una UBS, la cual no participó en el estudio; posteriormente se adecuaron los formularios, donde se recolectaron todos los datos de modo prospectivo. La investigadora principal realizó todas las entrevistas.

Indicadores de prescripción. a) Número promedio de medicamentos por prescripción; b) porcentaje de medicamentos prescritos por su denominación genérica; c) porcentaje de prescripciones que contenían antibióticos; d) porcentaje de prescripciones que contenían medicamentos inyectables, y e) porcentaje de prescripciones que contenían medicamentos de la REMUNE.

En las UBS de Londrina las prescripciones se registran en el expediente del paciente; en cada una se revisaron 30 historiales médicos con prescripción de medicamentos de pacientes atendidos el mismo día de la recolección de datos.

Para efectos de este estudio, se consideró como genérica la Denominación Común Brasileña (DCB) y en su ausencia, el International Nonproprietary Name (INN) (11). Asimismo, se consideraron antibióticos aquellos medicamentos que pertenecían a la clasificación de antiinfecciosos de la $12^{\mathrm{a}}$ lista de medicamentos esenciales de la OMS (12). Las vacunas no fueron consideradas en el cálculo del porcentaje de inyectables, según recomendación de la OMS (4). Para verificar si el medicamento otorgado constaba en la Relación Municipal de Medicamentos Esenciales (REMUME), se utilizó el listado proporcionado por la AMS.

Indicadores de atención al paciente. a) Tiempo promedio de consulta (minutos); b) tiempo promedio de dispensación de los medicamentos (segundos); c) porcentaje de medicamentos dispensados; d) porcentaje de medicamentos correctamente etiquetados, y e) porcentaje de pacientes con la información necesaria para usar correctamente los medicamentos.

La duración de la consulta se obtuvo cronometrando el tiempo entre la entrada y la salida del paciente de la consulta médica. Para verificar el conocimiento de los pacientes sobre el uso correcto de los medicamentos (el nombre del medicamento, la dosis, el intervalo y la duración del tratamiento), se realizaron entrevistas de los pacientes que recibieron prescripción a la salida de la unidad de salud.
Indicadores del servicio de salud. a) Existencia de una copia de la REMUME y b) disponibilidad de los medicamentos clave (para esta finalidad, se consideraron los medicamentos incluidos en la "Portaria 16/2000" del Ministerio de la Salud (13), de disponibilidad obligatoria en los servicios farmacéuticos de atención primaria).

\section{RESULTADOS}

Ninguna UBS del municipio contaba con un farmacéutico en el período de estudio; el personal de nivel técnico de enfermería (auxiliares), sin formación específica para el manejo de medicamentos, los entregaba a la población.

Indicadores de prescripción. Se encontró un promedio de 2,2 (Intervalo de confianza de 95\% [IC95\%] 1,7-2,6) medicamentos por prescripción. Se utilizó la denominación genérica en 66,5\% (IC95\% $44,9-82,5)$ de las prescripciones. El porcentaje de prescripciones de antibióticos fue de $28,6 \%$ (IC95\% 10-56,6). El uso de inyectables fue de 10, 9\% (IC95\% 3,3-30) en promedio. Del total de prescripciones, $73,3 \%$ (IC95\% 67,1-78,2) de los medicamentos constaban en la REMUME (cuadro 1).

Indicadores de atención al paciente. $\mathrm{El}$ tiempo de consulta promedio fue de 8,6 (IC95\% 3,9-12,5) minutos. En promedio, $71,1 \%$ (IC95\% 62,8-80,0) de los medicamentos prescritos fueron dispensados. Solamente 46,6\% (IC95\% 36,6-66,6) de pacientes reportaron poseer la información necesaria para usar correctamente los medicamentos.

Como los auxiliares entregan los medicamentos después de la consulta, al mismo tiempo que realizan otros procedimientos, no fue posible medir el tiempo de dispensación. Lo mismo aconteció con el indicador referente al Correcto etiquetaje de los medicamentos, debido a que las UBS no entregaban información escrita a los pacientes, por una falta de estandarización y uniformidad en el proceso de trabajo.

Indicadores del servicio de salud. La REMUME estaba disponible en $70 \%$ de las UBS y de estas, $23 \%$ poseían más de un ejemplar. Todas las UBS tenían por lo menos $80 \%$ de los medicamentos clave de la "Portaria 16/2000" del Ministerio de Salud; sin embargo, solamente $46,1 \%$ de las unidades contaban con todos los medicamentos.

En el cuadro 2 se presentan los resultados de este estudio, comparados con los resultados obtenidos mediante la aplicación de la misma metodología en otras regiones del país.

\section{DISCUSIÓN}

El promedio de medicamentos por consulta de 2,2 está en el límite máximo de lo considerado aceptable por la OMS, que es de 1,3 a 2,2 medicamentos por prescripción, aunque es un valor sin tendencia a la polifarmacia $(4,5)$. Otros trabajos llegaron a resultados semejantes (con variación de 1,7 a 3,1) $(14,15)$. En un estudio de la OMS, realizado en 12 países, el promedio tuvo una variación de 1,3 a 3,8 medicamentos por prescripción (5). El estudio nacional realizado en 2004 en el Brasil encontró resultados semejantes (2,3 medicamentos por prescripción) (9).

El grado de polifarmacia es un indicador indirecto de la calidad de la prescripción. Además, cuanto mayor el valor encontrado, se debe tener mayor cuidado en relación con la prescripción, la orientación y el seguimiento para preve-
CUADRO 1. Indicadores seleccionados del uso de medicamentos en
servicios farmacéuticos de atención primaria del Sistema Único de
Salud de Londrina, Paraná, Brasil, 2003

\begin{tabular}{lccc}
\hline \multicolumn{1}{c}{ Indicador } & Porcentaje & Mediana & $\begin{array}{c}\text { Desviación } \\
\text { estándar }\end{array}$ \\
\hline Medicamentos/consulta & $N^{\mathrm{a}}$ & 2,2 & 9,4 \\
Genéricos & 66,5 & 1,4 & 14,0 \\
Antibióticos & 28,6 & 0,6 & 9,7 \\
Injetáveis & 10,9 & 0,4 & 12,0 \\
Medicamentos REMUME & 73,3 & 1,6 & 6,7 \\
Tempo de consulta & $\mathrm{ND}^{\mathrm{a}}$ & 8,6 & 4,7 \\
Medicamentos dispensados & 71,1 & 1,5 & 6,7 \\
\hline${ }^{a}$ No disponible. & & &
\end{tabular}


CUADRO 2. Comparación entre indicadores seleccionados del uso de medicamentos obtenidos en estudios realizados en Brasil

\begin{tabular}{|c|c|c|c|c|c|c|c|c|}
\hline Indicador & $\begin{array}{c}\text { Fortaleza, }^{a} \\
\text { Ceará (a) }\end{array}$ & $\begin{array}{c}\text { Fortaleza, }^{\text {b }} \\
\text { Ceará (b) }\end{array}$ & $\begin{array}{c}\text { Salvador, } \\
\text { Bahia }\end{array}$ & $\begin{array}{c}\text { Interior, } \\
\text { Bahia }\end{array}$ & $\begin{array}{c}\text { Ribeirão } \\
\text { Preto, }^{\mathrm{d}} \\
\text { São } \\
\text { Paulo }\end{array}$ & $\begin{array}{c}\text { Campo } \\
\text { Grande, } \\
\text { Mato } \\
\text { Grosso } \\
\text { do Sul }\end{array}$ & $\begin{array}{c}\text { Brasília, }{ }^{\dagger} \\
\text { Distrito } \\
\text { Federal }\end{array}$ & $\begin{array}{l}\text { Londrina, }{ }^{g} \\
\text { Paraná }\end{array}$ \\
\hline No. de medicamentos/prescripción & 3,1 & 2,2 & 1,7 & 1,8 & 2,4 & 2,3 & 2,3 & 2,2 \\
\hline Prescripción del nombre genérico (\%) & 84,0 & 74,0 & 44,5 & 44,7 & 30,5 & 84,3 & 73,2 & 66,5 \\
\hline Prescripción de antibióticos (\%) & 19,0 & 37,0 & 38,1 & 32,5 & 21,3 & 27,4 & 26,4 & 28,6 \\
\hline Prescripción de inyectables (\%) & - & 11,0 & 2,2 & 4,9 & 8,2 & 10,2 & 7,6 & 10,9 \\
\hline Prescripción de edicamentos REMUME (\%) & 96,0 & 78,0 & 68,0 & 64,5 & 80,1 & 92,7 & 85,3 & 73,3 \\
\hline Tiempo de consulta (minutos) & 8,6 & 5,8 & 6,4 & 5,3 & 9,1 & 5,5 & 9,4 & 8,6 \\
\hline Tiempo de dispensación (segundos) & 10,2 & 17,0 & 44,0 & 41,0 & 18,3 & 55,0 & 53,9 & - \\
\hline Medicamentos dispensados (\%) & 84 & 66,0 & 39,5 & 81,4 & 60,3 & 80,7 & 61,2 & 71,1 \\
\hline Conocimiento/ uso correto (\%) & 43,0 & 54,0 & - & - & 70,0 & 56,7 & 18,7 & 46,6 \\
\hline Prescripción de medicamentos clave (\%) & - & - & - & - & 98,4 & 87,2 & 83,2 & - \\
\hline
\end{tabular}

Fuentes:

a Teixeira ACA, Serra GMC, Silva JA. Perfil da utilização de medicamentos na unidade de pacientes externos do Hospital Mesejana.-CE. En: Seminário Brasileiro de Farmacoepidemiologia. Fortaleza; Livro de resumos. Fortaleza: EUFC; 1996.

b Lopes AEC, Teixeira ACA, Gurgel MLF; Miranda MCC, Oliveira MA, Oliveira MML, Murta RLG. Drug use of evaluation in health services in Fortaleza, Brazil. INRUD News. 1996;(6):16.

c Pacheco F, Aguiar MGG, Queiroz AM. Diagnóstico da assistência farmacêutica do estado da Bahia. Salvador: Secretaria de Estado da Saúde, 1998.

d Santos V, Nitrini SMOO. Indicadores do uso de medicamentos prescritos e de assistência ao paciente de serviços de saúde. Rev Saúde Pública. 2004;38(6):819-34.

e Cunha MCN, Zorzatto JR, Castro LLC. Avaliação do uso de medicamentos na Rede Pública Municipal de Campo Grande-MS. Revista Ciências Farmacêuticas. 2002;38(2):216-27.

${ }^{\dagger}$ Naves JOS, Silver LD. Avaliação da assistência farmacêutica na atenção primária no Distrito Federal. Rev Saúde Pública 2005;39(2):223-30.

${ }^{g}$ Resultados del presente trabajo.

nir problemas relacionados con medicamentos, tales como interacciones no deseadas, confusiones con el régimen de tratamiento y cambio de medicamentos, entre otros.

La prescripción de 66,5\% de los medicamentos por su denominación genérica es un resultado bajo, comparado con otros trabajos $(3,14,15,16)$, sobre todo porque, además de ser una recomendación internacional (17), es obligatoria en el SUS $(8,11)$.

El porcentaje de $28,6 \%$ de prescripciones de antibióticos está muy próximo a la de otros trabajos (cuadro 2) y dentro del rango recomendado (de 20 a 30\%) (5). Sin embargo, al considerarse individualmente, en tres UBS se encontraron valores mayores de lo recomendado $(40,0 \%$, $56,6 \%$ y $33,3 \%$ ). Esto puede estar relacionado al brote de conjuntivitis viral que ocurrió en el sur del Brasil a principios de 2003: desde el final de febrero hasta el 26 de marzo, el Estado de Paraná registró 15395 casos de conjuntivitis viral (18). La AMS de Londrina adoptó el uso de colirio de gentamicina como protocolo para prevenir infecciones bacterianas concomitantes, por lo que este medicamento fue indicado a todos los pacientes con conjuntivitis, lo que provocó una gran diferencia en el cálculo del indicador prescripción de antibióticos.

Las consecuencias del uso inapropiado de los antibióticos pueden crear graves problemas en el control de infecciones, a nivel individual y colectivo. Con- siderando que el uso de antibióticos genera resistencia, lo mejor es evitar el uso abusivo e inapropiado de estos fármacos (19).

El uso de inyectables no se justifica cuando están disponibles medicamentos con formas de administración menos invasivas, sobre todo en atención primaria (20). Sin embargo, los medicamentos inyectables están dentro de lo recomendado por la International Network for the Rational Use of Drug (INRUD) (5), lo que determina que su prescripción tenga valores hasta de $20 \%$. En comparación con otros trabajos realizados, está entre los promedios más altos $(3,15,16,21)$.

La prescripción de medicamentos presentes en la REMUME fue semejante a la de otros trabajos $(16,21,22)$. Dos factores pueden explicar el hecho de que $26,7 \%$ de los medicamentos prescritos no eran de la REMUME: posibles problemas en la selección, lo que indicaría la necesidad de ajuste de la REMUME al perfil epidemiológico, o la elección por el médico de un medicamento no estandarizado para una determinada patología, que podría ser tratada con uno de la lista. En este sentido, hay que resaltar que la selección de medicamentos esenciales ofrece ventajas, tales como la reducción del número de productos farmacéuticos adquiridos, analizados, almacenados y distribuidos. Además, contribuye para mejorar el empleo, la gestión, la información y la vigilancia de los medicamentos y es un estímulo a las industrias locales $(23,24)$.
El tiempo promedio de consulta fue de ocho minutos. Aunque es un valor intermedio comparado con otros trabajos (3, $14,15,16,21,22)$, la legislación brasileña recomienda que en el SUS las consultas tengan una duración de 15 minutos (25), que es el tiempo recomendado por la OMS (4). El INRUD no indica un valor óptimo y se limita a comentar que un tiempo muy breve por consulta y dispensación refleja la posible existencia de algún problema, y un tiempo muy largo no añade información precisa. Según Starfield (26), es probable que en la práctica los pacientes no puedan hablar sobre su problema tanto tiempo como les gustaría, ya sea porque el profesional dirige el flujo de la comunicación, o porque el paciente no dispone de tiempo suficiente. Una buena relación médicopaciente se establece por medio del respeto a los sentimientos y puntos de vista del paciente, comprensión y disposición para establecer una relación que permita que el paciente sea corresponsable por los resultados del tratamiento (27); es decir, se requiere tiempo para que los médicos y pacientes establezcan una relación de confianza mutua.

El porcentaje de $71,1 \%$ de medicamentos prescritos que fueron dispensados es semejante a lo encontrado en otros estudios $(3,14,15,16,21,22)$. Hubo una discreta diferencia entre los medicamentos prescritos que pertenecen a la REMUME y los dispensados en las UBS, lo que puede ser una señal de pequeños proble- 
mas de programación o distribución de los medicamentos de la REMUME.

Llama la atención el hecho de que menos de la mitad de los pacientes $(46,6 \%)$ dijeron poseer la información necesaria para usar correctamente los medicamentos, aunque otros dos estudios han encontrado promedios aún más bajos $(14,15)$. Este indicador refleja, de cierta manera, la calidad de la información y de la atención recibida. Conforme a la ley, la prescripción debe ser expresa por medio de una receta (8); sin embrago, el hecho de que en este municipio algunos pacientes no reciban la receta médica escrita $-y$ de que los medicamentos sean entregados por personal sin entrenamiento específicodisminuye aún más las fuentes de información y representa una de las principales barreras para el uso racional de los medicamentos. Todo esto sugiere que, aunque los medicamentos están disponibles, es posible que los pacientes no los utilicen adecuadamente por falta de información y orientación, lo que aumenta aún más los riesgos del uso ya que se observó que el servicio de salud no ofrecía ningún tipo de seguimiento posterior a la entrega del medicamento. Además, aunque muchos factores influyen en la falta de adhesión al tratamiento, ninguno es tan determinante como el incumplimiento de las instrucciones sobre el tratamiento por la falta de información (28).
En una investigación posterior a la consulta ambulatoria en el Hospital Universitario de la ciudad de Porto Alegre, en Rio Grande do Sul, realizada con los mismos criterios, preguntas y clasificación de las respuestas que este estudio, se demostró que apenas $34 \%$ de los pacientes tenían un buen nivel de conocimientos sobre los medicamentos recibidos (29), porcentaje aún menor que el resultado aquí encontrado.

La REMUME debería estar disponible en todas las unidades, para los médicos, los pacientes y otros profesionales de salud (5), así como todos los medicamentos de la "Portaria 16/2000". El hecho de que no estuvieran presentes todos los medicamentos obligatorios sugiere posibles problemas de desabastecimiento relacionados a la programación, adquisición o distribución de los medicamentos.

La reestructuración de los servicios farmacéuticos es el punto clave para que los municipios logren mayor racionalidad y capacidad resolutiva en la utilización de los medicamentos y de los recursos del SUS. Para la gestión y la atención del paciente en los servicios farmacéuticos se necesitan recursos humanos capacitados y suficientes; de esta forma se puede garantizar la calidad en todos los procesos, para evitar el desabastecimiento, el desperdicio y promover el acceso y la seguridad del paciente. Es imprescindible superar el concepto de medicamento como producto, para verlo como recurso terapéutico necesario para apoyar a las demás acciones de salud.

\section{Conclusiones}

La adopción de indicadores es una herramienta importante para la toma de decisiones en la gestión y para la evaluación de las políticas farmacéuticas; la realización de estudios como este puede servir de línea de base para la evaluación de intervenciones. Aunque la metodología tiene limitaciones, su ventaja es que al ser estandarizada y haber sido validada, permite comparar los datos a otras realidades y en un mismo sitio en diferentes intervalos de tiempo.

La descentralización de la "Assistência Farmacêutica" y su organización como parte de los servicios de salud son recientes comparadas a todos componentes de la atención a la salud. El estudio demuestra que aunque se ha avanzado mucho en la gestión de los medicamentos con el fortalecimiento del acceso, es importante mirar hacia las etapas relacionadas con su utilización, en el sentido de promover un uso racional. Tal realidad es muy semejante a otros municipios del país. Aunque el Ministerio de Salud, los Estados y los municipios han realizado varias intervenciones en años recientes, la promoción del uso racional de medicamentos y la mejoría de la calidad de los servicios forman parte de una agenda inconclusa.

\section{REFERENCIAS}

1. Hernández PJS. Monitorización del uso de medicamentos para mejorar la calidad de la prescripción. Problemas, métodos e indicadores. Atención Primaria. 1998;18(6):331-8.

2. World Health Organization. WHO Medicines Strategy: countries at the core. 2004-2007. Ginebra: WHO; 2004.

3. Cunha MCN, Zorzatto JR, Castro LLC. Avaliação do uso de medicamentos na Rede Pública Municipal de Campo Grande-MS. Revista Ciências Farmacêuticas. 2002;38(2): 216-27.

4. Organización Mundial de la Salud. Como investigar el uso de medicamentos en los servicios de salud: Indicadores seleccionados del uso de medicamentos. Ginebra: OMS; 1993. [DAP. 93.1:1993].

5. Hogerzeil HV, Ross-Degnan D, Lang RO, Ofori-Adjei D, Santoso B, Chowdhury AK et al. Field tests for rational drug use in twelve developing countries. Lancet. 1993; 342:1408-10.
6. Brasil, Constituição da República Federativa do Brasil. Promulgada em 05 de outubro de 1988. Coleção Saraiva de Legislação. São Paulo: Saraiva;1995.

7. Bermudez, JZ. Indústria farmacêutica, estado e sociedade. São Paulo: Hucitec; Sobravime; 1995. 204p.

8. Brasil, Ministério da Saúde. Assistência Farmacêutica: instruções técnicas para sua organização. Brasília: Ministério da Saúde; 2001: $113 \mathrm{p}$.

9. Organização Pan-Americana da Saúde/Organização Mundial da Saúde; Brasil, Ministério da Saúde. Avaliação da Assistência Farmacêutica: estrutura, processo e resultados. Brasília: OPAS/OMS; Ministério da Saúde; 2005. 260p.

10. Brasil, Londrina. Prefeitura do Município. Características gerais do Município. Disponible en: http:/ /arara.londrina.pr.gov.br/ planejamento/perfil/perfil2004/. Acceso el 20 de mayo de 2006.
11. Brasil. Lei $\mathrm{n}^{\mathrm{0} 9787}$ de 10 de fevereiro de 1999. Lei dos os medicamentos genéricos e dá outras providências. Brasília: Diário Oficial da União, 11 de fevereiro 1999b.

12. Organización Mundial de la Salud. La $12^{\text {a }}$ lista modelo de medicamentos esenciales. Ginebra: OMS; 2002. Disponible en: http:// whqlibdoc.who.int/hq/2002/a76884.pdf. Acceso el 09 de marzo de 2009.

13. Brasil, Ministério da Saúde, Secretaria de Políticas de Saúde, Departamento de Atenção Básica, Gerência Técnica de Assistência Farmacêutica. Incentivo à Assistência Farmacêutica Básica: o que é e como funciona. Brasília: Ministério da Saúde; 2001. Disponible en: http:/ / bvsms.saude.gov.br/bvs/publica coes/incentivo_assit_farm.pdf. Acceso el 09 de marzo de 2009.

14. Teixeira ACA, Serra GMC, Silva JA. Perfil da utilização de medicamentos na unidade de pacientes externos do Hospital Mesejana.-CE. En: Seminário Brasileiro de Farmacoepide- 
miologia. Fortaleza; Livro de resumos. Fortaleza: EUFC; 1996.

15. Naves JOS, Silver LD. Avaliação da assistência farmacêutica na atenção primária no Distrito Federal. Rev Saúde Pública 2005;39(2): 223-30.

16. Lopes AEC, Teixeira ACA, Gurgel MLF; Miranda MCC, Oliveira MA, Oliveira MML, Murta RLG. Drug use of evaluation in health services in Fortaleza, Brazil. INRUD News. 1996;(6):16.

17. World Health Organization. Indicators for monitoring National Drug Policies. Ginebra: Action Programme on Essential Drugs; 1994.

18. Brasil, Fundação Nacional de Saúde (FUNASA). Surto de conjuntivite no sul do Brasil; 2003. Disponible en: www.funasa.gov.br/ Web\%20Funasa/not/not2003/not401.htm. Acceso el 09 de marzo de 2009.

19. Wannmacher L. Uso indiscriminado de antibióticos e resistência microbiana: Uma guerra perdida? Uso Racional de Medicamentos: temas selecionados. 2004; 1(4): 6p. Disponible en: http://www.opas.org.br/medicamentos/
site/UploadArq/HSE_URM_ATB_0304.pdf. Acceso: 2 de diciembre de 2005.

20. Hofler R, Silva EV, Vidotti C. Antigripais injetáveis no Brasil. Farmacoterapêutica: Boletim do Centro de Informações sobre Medicamentos. Brasília, n.5 ano 3., set/out. 1998.

21. Santos V, Nitrini SMOO. Indicadores do uso de medicamentos prescritos e de assistência ao paciente de serviços de saúde. Rev Saúde Pública. 2004;38(6):819-34.

22. Pacheco F, Aguiar MGG, Queiroz AM. Diagnóstico da assistência farmacêutica do estado da Bahia. Salvador: Secretaria de Estado da Saúde, 1998.

23. Dupim JAA, Righi RE. Medicamentos essenciais nos sistemas locais de saúde. En: Bonfim JRA, Mercucci VL, (org.). A construção da política de medicamentos. São Paulo: Ed. Hucitec SOBRAVIME; 1997.

24. Marin N, Luiza VL, Osorio-de-Castro CGS, Machado-dos-Santos S. Assistência farmacêutica para gerentes municipais. Brasília: OPAS/OMS, 2003. Disponible en: http:// portal.saude.gov.br/portal/arquivos/pdf/af_ gerentes_municipais.pdf. Acceso: 20 de enero de 2004.

25. Brasil, Ministério da Saúde. Portaria GM no 1.101 de 12 de junho de 2002. Parâmetros de cobertura assistencial no âmbito do Sistema Único de Saúde - SUS. Brasília: Ministério da Saúde; 2002.

26. Starfield, B. Atenção Primária: equilíbrio entre necessidades de saúde, serviços e tecnologia. Brasília, UNESCO; 2002.

27. Organização Mundial da Saúde. Guia para Boa Prescrição Médica. Porto Alegre: ARTMED; 1998.

28. World Health Organization. Adherence to long-term therapies. Geneva: WHO; 2003.

29. Silva T, Schenkel EP, Mengue SS. Nível de informação a respeito de medicamentos prescritos a pacientes ambulatoriais de hospital universitário. Caderno Saúde Pública. 2000; 16(2):449-55.

Manuscrito recibido el 28 de junio de 2007. Aceptado para publicación, tras revisión, el 4 de agosto de 2008.
ABSTRACT

Description of the pharmaceutical services offered by the universal health care system at the primary care level in Londrina, Paraná, Brazil

Key words
Objectives. To describe the pharmaceutical services offered at the primary care level by Brazil's Sistema Único de Salud (Universal Health Care System).

Methods. The study took place in Londrina, Paraná, in January-March 2003. The World Health Organization's recommended drug use indicators were employed. Included in the study were 13 primary health care facilities in which 390 patients were interviewed.

Results. None of the facilities had a pharmacist. The average office visit lasted 8.6 minutes; the mean number of drugs prescribed was 2.2; and injections were given in $10.9 \%$. Generic drugs were prescribed $66.5 \%$ of the time and $73 \%$ of the prescribed drugs were included in the Lista Municipal de Medicamentos Esenciales (Municipal List of Essential Drugs). Only $46.6 \%$ of the patients interviewed reported having the basic instructions required for appropriate use of the drug prescribed.

Conclusions. These results can aid in restructuring the municipality's pharmaceutical services because they underscore the need to improve and to increase rational drug use as a therapeutic resource imperative to supporting health efforts.

Drugs; pharmacoepidemiology; pharmaceutical services; drugs, essential; primary care; Brazil. 